\title{
Imaging the Parasinus Region with a Third-Generation Dual-Source CT and the Effect of Tin Filtration on Image Quality and Radiation Dose
}

\author{
M.M. Lell, M.S. May, M. Brand, A. Eller, T. Buder, E. Hofmann, M. Uder, and W. Wuest
}

\begin{abstract}
BACKGROUND AND PURPOSE: CT is the imaging technique of choice in the evaluation of midface trauma or inflammatory disease. We performed a systematic evaluation of scan protocols to optimize image quality and radiation exposure on third-generation dual-source CT.
\end{abstract}

MATERIALS AND METHODS: CT protocols with different tube voltage (70-150 kV), current (25-300 reference mAs), prefiltration, pitch value, and rotation time were systematically evaluated. All images were reconstructed with iterative reconstruction (Advanced Modeled Iterative Reconstruction, level 2). To individually compare results with otherwise identical factors, we obtained all scans on a frozen human head. Conebeam CT was performed for image quality and dose comparison with multidetector row CT. Delineation of important anatomic structures and incidental pathologic conditions in the cadaver head was evaluated.

RESULTS: One hundred kilovolts with tin prefiltration demonstrated the best compromise between dose and image quality. The most dose-effective combination for trauma imaging was Sn100 kV/250 mAs (volume CT dose index, 2.02 mGy), and for preoperative sinus surgery planning, Sn100 kV/150 mAs (volume CT dose index, $1.22 \mathrm{mGy}$ ). "Sn" indicates an additional prefiltration of the x-ray beam with a tin filter to constrict the energy spectrum. Exclusion of sinonasal disease was possible with even a lower dose by using Sn100 kV/25 mAs (volume CT dose index, $0.2 \mathrm{mGy}$ ).

CONCLUSIONS: High image quality at very low dose levels can be achieved by using a Sn100-kV protocol with iterative reconstruction. The effective dose is comparable with that of conventional radiography, and the high image quality at even lower radiation exposure favors multidetector row CT over conebeam CT.

ABBREVIATIONS: $\mathrm{CBCT}=$ conebeam $\mathrm{CT} ; \mathrm{CTDI}_{\text {vol }}=$ volume $\mathrm{CT}$ dose index; $\mathrm{HPM}=$ high-pitch mode; $\mathrm{MDCT}=$ multidetector row $\mathrm{CT} ; \mathrm{Sn}=$ an additional prefiltration of the $x$-ray beam with a tin filter to constrict the energy spectrum

$\mathbf{P}$ rojection radiography was used in the past for the evaluation of midface trauma and inflammatory sinonasal disease, but due to insufficient delineation of the complex anatomy, crosssectional imaging has largely replaced radiography. ${ }^{1-4}$ Multidetector row CT (MDCT) has become the criterion standard due to its exquisite delineation of small bony details, 3D properties, short examination time, and relatively low cost. MDCT is, however, responsible for the most radiation exposure in medical imaging. MR imaging can also exquisitely depict sinonasal mucosal disease, thereby avoiding radiation exposure, but delineation of fine bony

Received August 15, 2014; accepted after revision December 14

From the Departments of Radiology (M.M.L., M.S.M., M.B., A.E., M.U., W.W.) and Orthodontics and Orofacial Orthopedics (E.H.); Department I (T.B.), Institute of Anatomy; and Imaging Science Institute (M.M.L., M.U.), University Erlangen, Erlangen, Germany.

Please address correspondence to Michael M. Lell, MD, Department of Radiology, University of Erlangen, Maximiliansplatz 1, 91054 Erlangen, Germany; e-mail: michael.lell@uk-erlangen.de

http://dx.doi.org/10.3174/ajnr.A4270 structures is not optimal. Conebeam CT (CBCT), primarily introduced for dental applications, has evolved as an alternative to MDCT because its large flat panel detectors can image the maxillofacial region or even the complete head and provide excellent detail of bony anatomy, though only very limited soft-tissue information. CBCT provides high spatial resolution and low radiation exposure, though at the cost of longer image-acquisition times and, therefore, a higher risk of motion artifacts. In recent years, radiation dose-optimized MDCT protocols have been proposed, reducing the tube current from 170 effective mAs at $120 \mathrm{kV}$ down to 33 effective mAs. ${ }^{5}$ Iterative reconstruction and special scanning techniques like high-pitch mode (HPM) and low-kilovolt scanning have further decreased radiation exposure. ${ }^{4,6,7} \mathrm{Ra}$ diation exposure is considered a relevant issue because sinonasal disease may require repetitive imaging in a relatively young, otherwise healthy patient population with radiosensitive organs within the field of direct exposure (ie, eye lenses) or scattered $\mathrm{x}$-rays (ie, thyroid gland).

Image quality and radiation exposure should be individually 
adapted to the specific clinical situations: While a high noise level may be acceptable in ruling out mucosal inflammation, less noise may be desired in the preoperative setting, where anatomic variants need to be ruled out and thin bony structures like the lamina papyracea and cribrosa must be assessed, especially if image-based navigation or robotic surgery is performed. ${ }^{8}$ Even higher image quality and therefore less noise are required for the assessment of trauma.

The aim of our study was to assess the image quality and radiation exposure of different MDCT protocols and to identify protocols with an optimal compromise between radiation exposure and image quality for specific indications.

\section{MATERIALS AND METHODS}

\section{MDCT Image Acquisition and Reconstruction}

The scans were performed on a cadaver head (see below) on a third-generation dual-source CT system (Somatom Definition Force; Siemens, Erlangen, Germany) in single-source helical mode with a detector collimation of $192 \times 0.6 \mathrm{~mm}$. Examinations were conducted with 5 different settings of the tube current (25, $50,100,150,200 \mathrm{mAs})$ and 7 different tube-voltage settings (70, 80, 90, 100, Sn100, 120, Sn150 kV). "Sn" indicates an additional prefiltration of the $\mathrm{x}$-ray beam with a tin filter to constrict the energy spectrum. Additional scans with 250 and $300 \mathrm{mAs}$ were performed with $\mathrm{Sn} 100 \mathrm{kV}$. Pitch values of 0.75 and 1.2 and gantry rotation times of 0.5 and 1.0 seconds were used, resulting in 148 different scan protocols.

All images were reconstructed with $0.6-\mathrm{mm}$ section thickness, 0.4-mm section interval, FOV $=210 \mathrm{~mm}$, sharp (bone) kernel (Hr64; Br64 in case of $\mathrm{Sn} 100 \mathrm{kV}$ ), and iterative reconstruction (Advanced Modeled Iterative Reconstruction, level 2).

\section{Conebeam CT}

A clinical flat panel conebeam CT system (ProMax 3D; Planmeca, Helsinki, Finland) with a maximum FOV $=230 \times 260 \mathrm{~mm}$ was used for comparison of image quality and radiation exposure. We used 2 clinical protocols: a high-resolution sinus protocol with a section thickness of $0.2 \mathrm{~mm}, 11 \mathrm{~mA}$ at $96 \mathrm{kV}$, and an $\mathrm{FOV}_{\mathrm{xy}}$ of 130 $\mathrm{mm}, \mathrm{FOV}_{\mathrm{z}}$ of $160 \mathrm{~mm}$, exposure time of 12.3 seconds; and a low-dose head protocol with a section thickness of $0.4 \mathrm{~mm}, 11 \mathrm{~mA}$ at $96 \mathrm{kV}$, an $\mathrm{FOV}_{\mathrm{xy}}$ of $230 \mathrm{~mm}, \mathrm{FOV}_{\mathrm{z}}$ of $160 \mathrm{~mm}$, exposure time 9.4 seconds.

\section{Data Transfer and Image-Quality Assessment}

The volume datasets were stored in DICOM format in our PACS system. A 3D postprocessing platform (syngo.via; Siemens) was used to display all datasets in MPR mode (1.5-mm thick sections, window-level setting of $3300 / 300 \mathrm{HU}$ on two 21.2-inch $(53.85$ $\mathrm{cm}$ ) high-resolution monitors (Radiforce RX 320; EIZO, Ishikawa, Japan) after removing all scan protocol-related information. The readers were allowed to adjust the window-level settings at their discretion. The datasets were analyzed by 2 readers with $>10$ years' experience in head and neck radiology.

\section{Scan Object}

A fresh frozen cadaver head of a white male adult was used for imaging. Ten anatomic regions (lamina papyracea, lamina cri- brosa, nasal septum, ethmoid air cells, sinus walls, orbital floor, lacrimal duct, temporomandibular joint, tympanic cavity, mastoid cells); and 5 incidental "pathologic" conditions (calcified ICA plaque, temporomandibular joint osteoarthritis, intracranial air collections, paranasal and mastoid fluid collections) were evaluated with a 5-point Likert scale: 5 indicating excellent, 4, good; 3 , moderate; 2 , sufficient; 1 , insufficient image quality. The overall rating for each protocol was defined by the worst rating of these 15 structures or findings. For trauma assessment, high image quality (score $\geq 4$ ) was required. Image quality of $\geq 3$ was considered sufficient for the preoperative evaluation for sinusitis surgery, and image quality of $\geq 2$, to detect or rule out sinusitis was considered acceptable. We did not perform noise measurements because noise values do not adequately reflect the diagnostic value in highcontrast objects.

To investigate the effects of different pitch values on image quality, we performed 1-to-1 comparisons of datasets after removing all identifying information, and data were displayed side by side in random order. The same procedure was performed for the different gantry-rotation times.

\section{Estimation of Radiation Exposure}

The estimation of the effective radiation dose (E) of the CT examination was based on the volume CT dose index $\left(\mathrm{CTDI}_{\mathrm{vol}}\right)$ and the dose-length product derived from the patient protocol with use of the specific conversion coefficient: $\mathrm{E}=(\mathrm{mGy} \times \mathrm{cm}) \times 0.0019$ $\left(\mathrm{mSv} \times \mathrm{mGy}^{-1} \times \mathrm{cm}^{-1}\right){ }^{9}$ In contrast to all other scans, the $\mathrm{CTDI}_{\mathrm{vol}}$ in the patient protocol of the Sn100-kV scan is referenced to a $32-\mathrm{cm}$ phantom. An additional conversion factor of 2.5 was applied to compensate for the different reference phantoms. All $\mathrm{CTDI}_{\mathrm{vol}}$ values following are referring to the $16-\mathrm{cm}$ phantom.

\section{Statistical Analysis}

Values are given as mean $\pm \mathrm{SD}$. The image quality was measured by using an ordinal performance scale with 5 levels. Interrater agreement was assessed by using the Cohen weighted $\kappa$ test. Fleiss equally arbitrary guidelines characterize a $\kappa$ of $>0.75$ as excellent, $0.40-0.75$ as fair to good, and $<0.40$ as poor. Significance levels of .05 were assumed. Statistical analysis was performed by using the software package SPSS Statistics, Version 19 (IBM, Armonk, New York).

\section{RESULTS}

The image quality differed significantly with dose. The readers did not detect significant differences in image quality between corresponding datasets acquired with a pitch value of 0.75 versus 1.2 or with a gantry-rotation time of 0.5 second versus 1 second.

Image quality for $S n 100 \mathrm{kV} / 300 \mathrm{mAs}$ and $250 \mathrm{mAs}$ was rated perfect (score of 5) by both readers, and the corresponding $\mathrm{CTDI}_{\mathrm{vol}}$ was 2.45 and $2.05 \mathrm{mGy}$. Image quality and the corresponding $\mathrm{CTDI}_{\mathrm{vol}}$ of the other tube current and tube voltage combinations are given in Tables 1 and 2.

Interrater agreement was good $(\kappa=0.71)$. The most doseeffective combinations for trauma imaging (requiring a rating of $\geq 4$ ) were Sn $100 \mathrm{kV} / 250 \mathrm{mAs}\left(\mathrm{CTDI}_{\mathrm{vol}}=2.05 \mathrm{mGy}\right)$, Sn150 $\mathrm{kV} / 50 \mathrm{mAs}\left(\mathrm{CTDI}_{\mathrm{vol}}=2.61 \mathrm{mGy}\right)$, and $90 \mathrm{kV} / 50 \mathrm{mAs}$ $\left(\mathrm{CTDI}_{\mathrm{vol}}=3.23 \mathrm{mGy}\right)$. 
Table 1: Scan protocols and image quality ${ }^{\mathrm{a}}$

\begin{tabular}{lccccc}
\hline & $\mathbf{2 0 0} \mathbf{~ m A s}$ & $\mathbf{1 5 0} \mathbf{m A s}$ & $\mathbf{1 0 0} \mathbf{m A s}$ & $\mathbf{5 0} \mathbf{m A s}$ & $\mathbf{2 5} \mathbf{~ m A s}$ \\
\hline $70 \mathrm{kV}$ & 4 & 3 & 3 & 2 & 2 \\
$80 \mathrm{kV}$ & 5 & 4 & 3 & 2 & 2 \\
$90 \mathrm{kV}$ & 5 & 5 & 5 & 4 & 3 \\
$100 \mathrm{kV}$ & 5 & 5 & 5 & 4 & 3 \\
$100 \mathrm{kV}+\mathrm{Sn}$ & 3 & 3 & 2 & 2 & 2 \\
$120 \mathrm{kV}$ & 5 & 5 & 5 & 4 & 3 \\
$150 \mathrm{kV}+\mathrm{Sn}$ & 5 & 5 & 5 & 4 & 3 \\
\hline
\end{tabular}

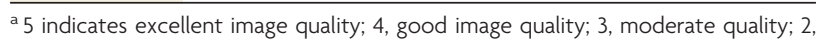
sufficient for special indications; 1 , insufficient.

Table 2: Scan protocols and $\mathrm{CTDI}_{\mathrm{vol}}{ }^{\mathrm{a}}$

\begin{tabular}{lccccc}
\hline & $\mathbf{2 0 0} \mathbf{~ m A s}$ & $\mathbf{1 5 0} \mathbf{~ m A s}$ & $\mathbf{1 0 0} \mathbf{m A s}$ & $\mathbf{5 0} \mathbf{~ m A s}$ & $\mathbf{2 5} \mathbf{~ m A s}$ \\
\hline $70 \mathrm{kV}$ & 5.71 & 4.27 & 2.84 & 1.41 & 0.71 \\
$80 \mathrm{kV}$ & 8.97 & 6.72 & 4.47 & 2.22 & 1.11 \\
$90 \mathrm{kV}$ & 13.05 & 9.78 & 6.50 & 3.23 & 1.62 \\
$100 \mathrm{kV}$ & 17.69 & 13.25 & 8.81 & 4.38 & 2.25 \\
$100 \mathrm{kV}+\mathrm{Sn}$ & 1.62 & 1.22 & 0.82 & 0.4 & 0.2 \\
$120 \mathrm{kV}$ & 28.41 & 21.28 & 14.16 & 7.03 & 3.61 \\
$150 \mathrm{kV}+\mathrm{Sn}$ & 10.56 & 7.91 & 5.26 & 2.61 & 1.34 \\
\hline
\end{tabular}

${ }^{\text {a }}$ All CTDI ${ }_{\text {vol }}$ values are converted to a 16-cm phantom.

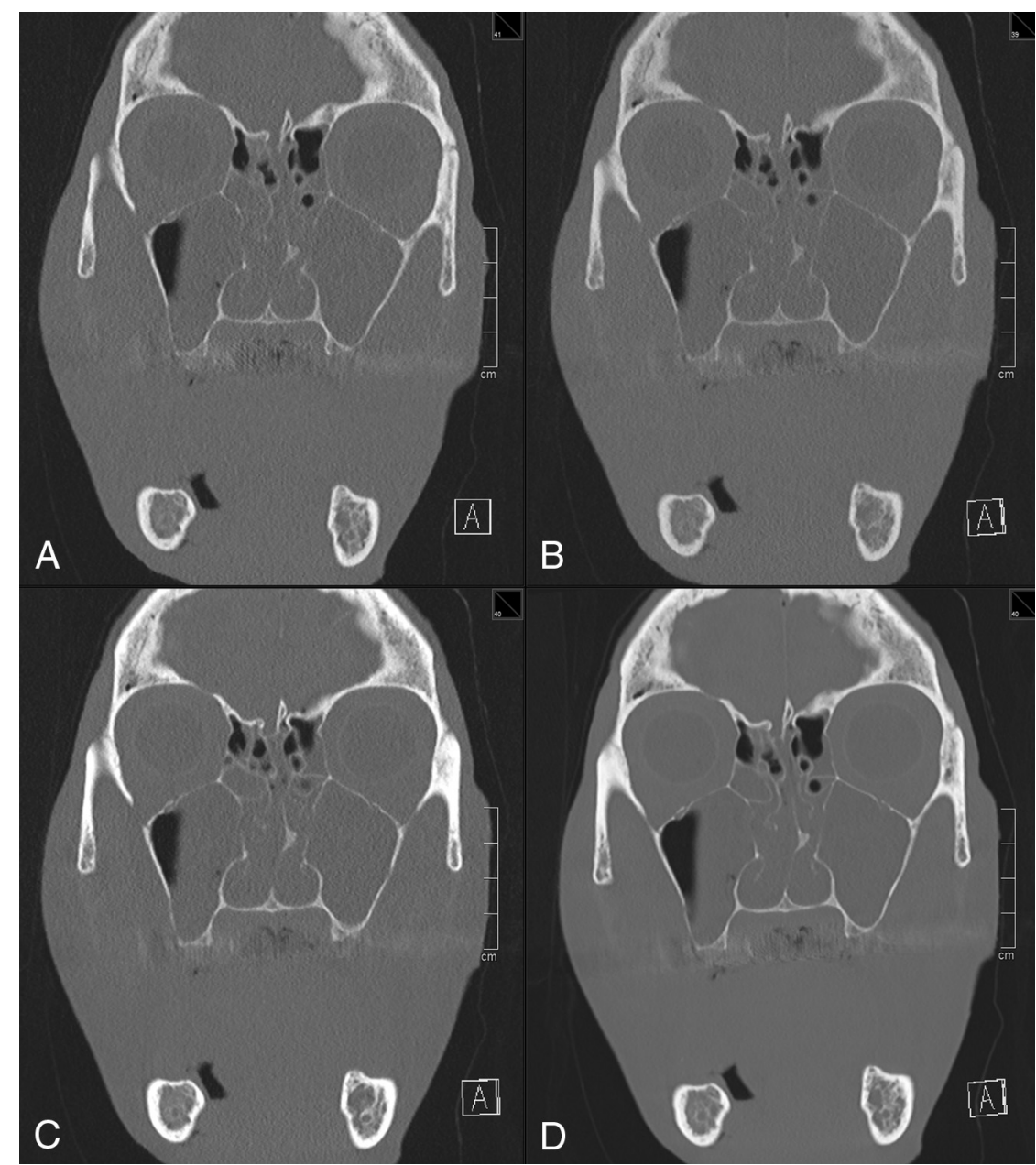

FIG 1. Dose-effective scan protocols achieving good image quality and high-dose reference protocol. The following image quality is considered sufficient for trauma imaging at the lowest dose. A, Sn100 kV/250 mAs. B, Sn150 kV/50 mAs. C, 90 kV/50 mAs. The following is a high-dose protocol. $D, 120 \mathrm{kV} / 200 \mathrm{mAs}$ is given as a reference. Coronal multiplanar reformation; section thickness, $1.5 \mathrm{~mm}$; window width-level, 3300/300 HU.
The most dose-effective combinations for preoperative sinus surgery planning (requiring a rating of $\geq 3$ ) were $\mathrm{Sn} 100 \mathrm{kV} / 150$ $\mathrm{mAs}\left(\mathrm{CTDI}_{\mathrm{vol}}=1.22 \mathrm{mGy}\right), \mathrm{Sn} 150 \mathrm{kV} / 25 \mathrm{mAs}\left(\mathrm{CTDI}_{\mathrm{vol}}=1.34\right.$ $\mathrm{mGy}$ ), $90 \mathrm{kV} / 25 \mathrm{mAs}\left(\mathrm{CTDI}_{\mathrm{vol}}=1.62 \mathrm{mGy}\right)$, and $100 \mathrm{kV} / 25 \mathrm{mAs}$ $\left(\mathrm{CTDI}_{\mathrm{vol}}=2.25 \mathrm{mGy}\right)$.

Both Sn100-kV and Sn150-kV protocols were associated with very low radiation exposure. The $\mathrm{Sn} 100-\mathrm{kV}$ protocols were associated with the lowest dose values, and high image quality sufficient for all indications was provided with tube currents of $300 \mathrm{mAs}$ $\left(\mathrm{CTDI}_{\mathrm{vol}}=2.45 \mathrm{mGy}\right)$ and $250 \mathrm{mAs}\left(\mathrm{CTDI}_{\mathrm{vol}}=2.05 \mathrm{mGy}\right)$. These scan protocols provide better image quality at a comparable dose level than a $100-\mathrm{kV}$ protocol without tin filtration and $25 \mathrm{mAs}$ (Fig 1). Image quality of $S n 100 \mathrm{kV}$ and $150 \mathrm{mAs}$ was sufficient for sinus surgery evaluation; the respective $\mathrm{CTDI}_{\mathrm{vol}}$ was $1.22 \mathrm{mGy}$, a dose level that could not be matched with other kilovolt settings (Fig 2).

CBCT image ratings were 4 (good) for an FOV of $130 \mathrm{~mm}$ and 3 (moderate) for an FOV of $230 \mathrm{~mm}$. The dose-area product was $2859 \mathrm{mGy} \times \mathrm{cm}^{2}$ and $2115 \mathrm{mGy} \times \mathrm{cm}^{2}$, respectively (Fig 3).

\section{DISCUSSION}

CT scanning of the sinuses is frequently performed; it has been estimated that approximately 4 million scans are obtained annually in the United States. ${ }^{10}$ Many of the patients are young, and examinations are often repeated. It is, therefore, mandatory to strictly follow the as low as reasonably achievable concept because the highly radiosensitive ocular lenses ${ }^{11}$ are within the scan range. Whether and to what extent there is a carcinogenic effect of very low radiation exposure is of current debate. ${ }^{12}$ Abundant data suggest that high image quality at low radiation exposure can be achieved with modern CT technology. Scanning at low-kilovolt settings results in a higher signal of iodine; thus, higher noise can be tolerated while maintaining the signal-to-noise ratio, contrast-to-noise ratio, and overall image quality. ${ }^{13,14}$ The dose-reduction potential is highest in CT angiography because of the high vascular attenuation. ${ }^{15-18}$ In nonenhanced CT, there is less potential of dose reduction by using low-kilovolt protocols, though the CT numbers of bone increase with decreasing kilovolt settings and the higher image noise in low kilovolts is a limiting factor. We demonstrated that the Sn100-kV protocol provided superior image quality compared with $70-$ to $90-\mathrm{kV}$ protocols at equivalent dose levels. This has also been reported in a phantom study of chest CT, where $\mathrm{Sn} 100 \mathrm{kV}$ was rated superior to 70 $\mathrm{kV}$ for nodule detection. ${ }^{19}$ Tin filtration restricts the bandwidth of the x-ray energy spectrum that reaches the patient. 


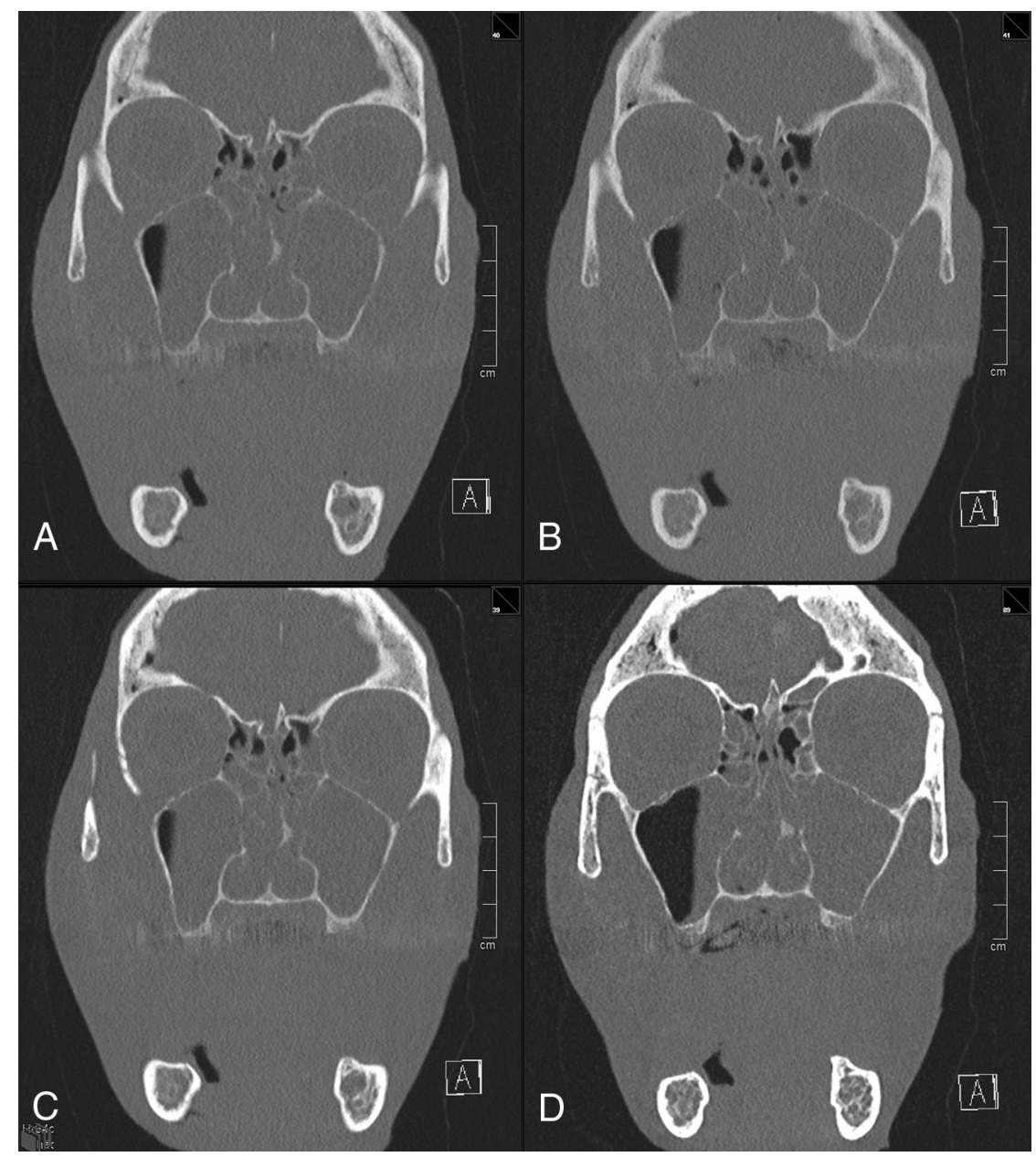

FIG 2. Dose-effective scan protocols achieving moderate image quality. The following image quality is considered sufficient for preoperative evaluation in noncomplicated sinusitis at the lowest dose. A, Sn100 kV/150 mAs. B, Sn150 kV/25 mAs. C, $90 \mathrm{kV} / 25 \mathrm{mAs}$. The following is a low-kilovolt protocol. $D, 70 \mathrm{kV} / 100 \mathrm{mAs}$ has higher attenuation values of bone but is associated with a higher dose. Coronal multiplanar reformation; section thickness, $1.5 \mathrm{~mm}$; window widthlevel, $3300 / 300 \mathrm{HU}$.

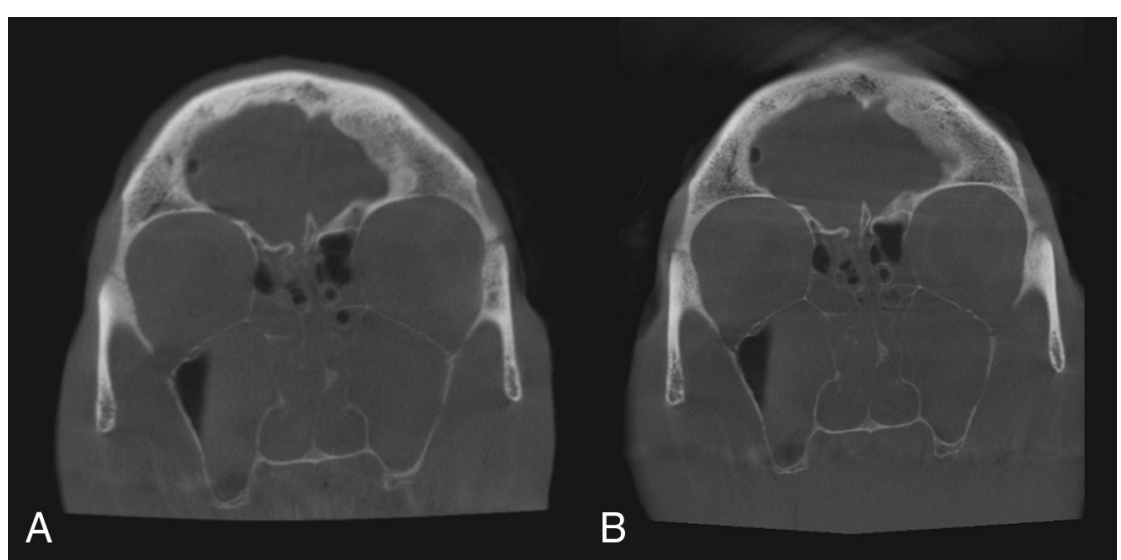

FIG 3. Conebeam CT with an FOV of $230 \mathrm{~mm}$ ( $A$, moderate image quality) and FOV of $130 \mathrm{~mm}$ ( $B$, good image quality). Note improvement of the delineation of bony structures with a smaller FOV and longer exposition time $(B)$.

The effective dose $(0.034 \mathrm{mSv}$ for a typical scan range of $10 \mathrm{~cm})$ is in the range of conventional projection $\mathrm{x}$-ray techniques (digital panoramic radiograph, $0.022 \mathrm{mSv}$ ) and below that of conebeam CT $(0.07-0.13 \mathrm{mSv}){ }^{20}$
Schulz et $\mathrm{al}^{7}$ proposed using an HPM $(P=3.0)$ to minimize radiation exposure. They reported an average dose at the eye lens of $0.64 \mathrm{mGy}$ and at the thyroid gland of $0.085 \mathrm{mGy}$. The same group published data of a patient cohort comparing conventional mode and HPM and stated that the effective dose may be reduced by half by using HPM, with a mean effective dose of $0.11 \mathrm{mSv} .^{21}$ Aksoy et $\mathrm{al}^{22}$ compared conventional mode and HPM on a second-generation dual-source CT scanner and considered HPM with a tube current of $18 \mathrm{mAs}$ at 80 $\mathrm{kV}$ sufficient to rule out sinus disease. The estimated effective dose was reported to be $0.02 \mathrm{mSv}$. Yang and Chen ${ }^{23}$ and Bulla et $\mathrm{al}^{24}$ criticized the reported dose values because the $\mathrm{CTDI}_{\mathrm{vol}}$ and dose-length product of HPM-a scan mode originally designed for cardiac and chest imaging - in the patient protocols of Siemens scanners are referenced to a $32-\mathrm{cm}$ phantom and not to a $16-\mathrm{cm}$ phantom, which is, by convention, used for head scans. To compensate for the difference, an additional conversion factor $(>2$, dependent on scanner and prefiltration) is necessary. In addition, dose distribution along the $\mathrm{z}$-axis is less homogeneous by using HPM, and over-ranging is higher compared with conventional pitch modes. ${ }^{16}$ This difference makes HPM less attractive for sinus imaging.

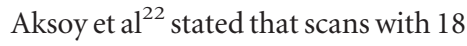
$\mathrm{mAs}$ at $80 \mathrm{kV}$ are sufficient to rule out sinusitis, but they did not recommend that protocol in the preoperative setting. This finding is in line with our findings in which $80 \mathrm{kV}$ and $25 \mathrm{mAs}$ were rated sufficient for special indications only (rule out sinusitis). We identified the most dose-effective protocol with sufficient image quality for preoperative planning to be $S n 100 \mathrm{kV}$ and $150 \mathrm{mAs}$, resulting in an effective dose of $0.03 \mathrm{mSv}$ for a typical $10-\mathrm{cm}$ scan range.

While CBCT is increasingly popular, MDCT systems provide much higher flexibility in data acquisition and image reconstruction. The scan range can be individually adapted, while only fixed FOVs are given in CBCT, leading to exposure of tissue not relevant for diagnosis. Image quality and also radiation exposure differ significantly among CBCT systems. ${ }^{25}$ While noise is relatively homogeneously distributed over the scan range in MDCT and can be balanced with tube current modula- 
tion and automatic exposure control algorithms, in CBCT, noise and reconstruction artifacts are unevenly distributed and increase from the central position toward the edges of the FOV. Iterative reconstruction algorithms can decrease overall image noise, but the appearance of the CT image may be altered by these algorithms. We used only a moderate setting of the iterative reconstruction algorithm in MDCT to avoid artifacts at delicate bone structures like the cribriform plate or the lamina papyracea.

In a recent study, MDCT and CBCT were performed on the same day for intraindividual comparison. ${ }^{26}$ Instead of using doseequivalent scan protocols, however, only "manufacturer recommended" protocols were applied; this application makes the comparison unbalanced in terms of dose and image quality. In another recent study, a comparison of MDCT and "simulated" CBCT was performed to evaluate the percentage of missed softtissue lesions, which was as low as 3.3\%. ${ }^{27}$ The authors used highdose MDCT examinations $\left(\mathrm{CTDI}_{\mathrm{vol}}=39.8-85.1 \mathrm{mGy}\right)$ as the reference and concluded that if one assumes appropriate selection of patients, CBCT can offer substantial radiation-dose reduction. These 2 reports are only the most recent ones of many suggesting that CBCT is more dose-efficient than MDCT. Two important conclusions can be drawn from these studies: First, in many imaging centers, low-dose MDCT protocols are still neglected, and second, appropriate patient selection is mandatory. We, therefore, propose different dose-optimized imaging protocols for specific indications in adults. Imaging of intracranial complications or tumor requires injection of contrast material, low noise levels, and therefore the use of standard dose protocols or MR imaging. For imaging the sinuses or midface trauma, the 3 most important tissue classes are bone, soft tissue, and air, which can be readily differentiated with low-dose protocols and do not necessitate contrast material injection. Still, image noise may not exceed a certain degree because otherwise, fissures, fracture lines, or a small dehiscence may not be detected. We found that $\mathrm{Sn} 100 \mathrm{kV} /$ $250 \mathrm{mAs}\left(\mathrm{CTDI}_{\mathrm{vol}}=2.02 \mathrm{mGy}\right)$ was the most dose-effective combination for trauma imaging. A little more noise could be tolerated in the presurgical evaluation of noncomplicated sinonasal inflammatory disease, but clear delineation of the cribriform plate, the lamina papyracea, the orbital walls, and neurovascular canals must be maintained. We identified Sn100 kV/150 mAs $\left(\mathrm{CTDI}_{\mathrm{vol}}=1.22 \mathrm{mGy}\right)$ as the protocol providing sufficient information at the lowest dose. To rule out mucosal swelling or fluid retention, protocols with even lower exposure $\left(\mathrm{CTDI}_{\mathrm{vol}}=0.2 \mathrm{mGy}\right)$ could be used, still providing non-superimposed 3D data.

Some limitations of our study need to be addressed. The evaluation of only 1 cadaveric head may be considered a limitation. However, for comparison of image quality, we consider the phantom having normal-sized sinus walls and extensive fluid collections within the sinuses more important than testing multiple phantoms. Additionally, we did not specify the organ dose of the thyroid and the eye lenses, yet with an effective dose of $0.03 \mathrm{mSv}$ for the parasinus region, eye lenses and thyroid exposure is an order of magnitude smaller than that with standard sinus protocols.

\section{CONCLUSIONS}

Our study demonstrates that imaging the paranasal sinuses for preoperative planning with a third-generation dual-source CT is feasible at very low radiation exposure $(0.03 \mathrm{mSv})$ when using $\mathrm{Sn} 100 \mathrm{kV}$ and iterative reconstruction. We optimized scan protocols for specific indications at the lowest possible dose and found that the radiation exposure is comparable with that of conventional radiography. Future studies in a large patient population are needed to confirm the clinical value of such protocols.

Disclosures: Michael M. Lell—UNRELATED: Grants/Grants Pending: Bayer*; Payment for Lectures (including service on Speakers Bureaus): Bayer, Bracco, Siemens. Matthias S. May —UNRELATED: Payment for Lectures (including service on Speakers Bureaus): Siemens. Michael Uder-UNRELATED: Payment for Lectures (including service on Speakers Bureaus): Bracco, Medtronic, Siemens, GE Healthcare. *Money paid to the institution.

\section{REFERENCES}

1. Kaur J, Chopra R. Three dimensional CT reconstruction for the evaluation and surgical planning of mid face fractures: a 100 case study. J Maxillofac Oral Surg 2010;9:323-28

2. Hopper RA, Salemy S, Sze RW. Diagnosis of midface fractures with CT: what the surgeon needs to know. Radiographics 2006;26:783-93

3. Duvoisin B, Landry M, Chapuis L, et al. Low-dose CT and inflammatory disease of the paranasal sinuses. Neuroradiology 1991;33: 403-06

4. Tack D, Widelec J, De Maertelaer V, et al. Comparison between lowdose and standard-dose multidetector CT in patients with suspected chronic sinusitis. AJR Am J Roentgenol 2003;181:939-44

5. Brem MH, Zamani AA, Riva R, et al. Multidetector CT of the paranasal sinus: potential for radiation dose reduction. Radiology 2007;243:847-52

6. Schulz B, Beeres M, Bodelle B, et al. Performance of iterative image reconstruction in $\mathrm{CT}$ of the paranasal sinuses: a phantom study. AJNR Am J Neuroradiol 2013;34:1072-76

7. Schulz B, Potente S, Zangos S, et al. Ultra-low dose dual-source highpitch computed tomography of the paranasal sinus: diagnostic sensitivity and radiation dose. Acta Radiol 2012;53:435-40

8. Bumm K, Wurm J, Rachinger J, et al. An automated robotic approach with redundant navigation for minimal invasive extended transsphenoidal skull base surgery. Minim Invasive Neurosurg 2005;48:159-64

9. Deak PD, Smal Y, Kalender WA. Multisection CT protocols: sex- and age-specific conversion factors used to determine effective dose from dose-length product. Radiology 2010;257:158-66

10. Stern SH, Spelic DC, Kaczmarek RV. NEXT 2000: protocol for survey of computed tomography (CT). December 18, 2000. http://www. crcpd.org/PDF/NEXT2000 CTProl.pdf. Accessed August 10, 2014

11. Stewart FA, Akleyev AV, Hauer-Jensen M, et al, on behalf of ICRP. ICRP publication 118: ICRP statement on tissue reactions and early and late effects of radiation in normal tissues and organs: threshold doses for tissue reactions in a radiation protection context. Ann ICRP 2012;41:1-322

12. Candela-Juan C, Montoro A, Ruiz-Martinez E, et al. Current knowledge on tumour induction by computed tomography should be carefully used. Eur Radiol 2014;24:649-56

13. Eller A, May MS, Scharf M, et al. Attenuation-based automatic kilovolt selection in abdominal computed tomography: effects on radiation exposure and image quality. Invest Radiol 2012;47:559-65

14. Eller A, Wuest W, Scharf M, et al. Attenuation-based automatic kilovolt (kV)-selection in computed tomography of the chest: effects on radiation exposure and image quality. Eur J Radiol 2013;82:2386-91

15. Eller A, Wuest W, Kramer M, et al. Carotid CTA: radiation exposure and image quality with the use of attenuation-based, automated kilovolt selection. AJNR Am J Neuroradiol 2014;35:237-41

16. Lell MM, May M, Deak P, et al. High-pitch spiral computed tomography: effect on image quality and radiation dose in pediatric chest computed tomography. Invest Radiol 2011;46:116-23

17. Winklehner A, Goetti R, Baumueller S, et al. Automated attenuationbased tube potential selection for thoracoabdominal computed to-

AJNR Am J Neuroradiol 36:1225-30 Jul 2015 www.ajnr.org

1229 
mography angiography: improved dose effectiveness. Invest Radiol 2011;46:767-73

18. Yu L, Fletcher JG, Grant KL, et al. Automatic selection of tube potential for radiation dose reduction in vascular and contrast-enhanced abdominopelvic CT. AJR Am J Roentgenol 2013;201:W297-306

19. Gordic S, Morsbach F, Schmidt B, et al. Ultralow-dose chest computed tomography for pulmonary nodule detection: first performance evaluation of single energy scanning with spectral shaping. Invest Radiol 2014;49:465-73

20. Grünheid T, Kolbeck Schieck JR, Pliska BT, et al. Dosimetry of a cone-beam computed tomography machine compared with a digital x-ray machine in orthodontic imaging. Am J Orthod Dentofacial Orthop 2012;141:436-43

21. Schell B, Bauer RW, Lehnert T, et al. Low-dose computed tomography of the paranasal sinus and facial skull using a high-pitch dualsource system: first clinical results. Eur Radiol 2011;21:107-12

22. Aksoy EA, Ozden SU, Karaarslan E, et al. Reliability of high-pitch ultra-low-dose paranasal sinus computed tomography for evaluating paranasal sinus anatomy and sinus disease. J Craniofac Surg 2014;25:1801-04
23. Yang WJ, Chen KM. Letter to the editor re: low-dose computed tomography of the paranasal sinus and facial skull using a high-pitch dual-source system-first clinical results. Eur Radiol 2011;21:1449_ 50; author reply 1451

24. Bulla S, Blanke P, Langer M, et al. Letter to the editor re: low-dose computed tomography of the paranasal sinus and facial skull using a high-pitch dual-source system-first clinical results. Eur Radiol 2011;21:1447-48; author reply 1451

25. Hofmann E, Schmid M, Sedlmair M, et al. Comparative study of image quality and radiation dose of cone beam and low-dose multislice computed tomography: an in-vitro investigation. Clin Oral Investig 2014;18:301-11

26. Leiva-Salinas C, Flors L, Gras P, et al. Dental flat panel CT in the evaluation of patients with inflammatory sinonasal disease: diagnostic efficacy and radiation dose savings. AJNR Am J Neuroradiol 2014;35:2052-57

27. Fakhran S, Alhilali L, Sreedher G, et al. Comparison of simulated cone beam computed tomography to conventional helical computed tomography for imaging of rhinosinusitis. Laryngoscope 2014;124:2002-06 